\section{Stimulus control and the growth of the infant's effective visual field*}

\author{
EDWARD TRONICK \\ Harvard University, Cambridge, Massachusetts 02138
}

The size of the infant's effective visual field was studied weekly in infants starting at 2 weeks of age until they were 10 weeks old. The field was initially quite small, $15 \mathrm{deg}$ to either side of their line of regard. Over the 2-month period of the study, it more than doubled for stimulus conditions in which the peripheral event was in motion and the fixation object static, and it remained approximately the same size when motion was present in the fixation event and the peripheral object was static. As with adults, the infant's effective visual field is directly related to the stimuli available.

The peripheral visual field registers information for the control of exploratory fixations of the environment and the registration of spatial information (Mackworth \& Morandi, 1967; Trevarthen, 1968). Limitations of the capacity for registration would have severe consequences for an organism's functioning. Objects and events exceeding the areal and temporal limits of the system would be unavailable, precluding actions related to them and deeper analysis by focal attentional processes. In a developing organism, a limited but gradually increasing capacity for registration could account for qualitative changes in behavior, such as the change from obligatory to active looking (Brennan et al, 1965), without requiring a developmental, structural change (Koffka, 1959; Aronson \& Tronick, 1970).

Previous assessments of the limits of the human infant's peripheral field found an initially limited range of effective vision that gradually increased with age (Peiper, 1963). The purpose of the following research was to quantify the growth of the infant's effective field of vision and to determine if these limits changed with changing stimulus conditions. To this end, infants were studied longitudinally in a visual field where their ability to register both static and dynamic visual events was assessed.

\section{METHOD \\ Subjects}

Eight infants, initially 2-3 weeks old, were used as Ss for 9 weekly sessions. All infants were clinically

\footnotetext{
* This research was supported by Grants MH-12623 from the National Institute of Mental Health and HD-03049 from the National Institute of Child Health and Human Development to Harvard University. Center for Cognitive Studies.

+Maiking address: Children's Hospital Medical Center, 300 Longwood Avenue, Boston, Massachusetts 02115 .
}

normal, and sex was not balanced for in the different experimental assignments. No cross-sectional group was examined, since the limited experience in the experiment-less than $20 \mathrm{~min}$ once a week-was thought to be insignificant, given the nonexperimental visual experience of the infants.

\section{Apparatus}

A semicircular infant table with a 20 -in. radius and 16 -in. legs was mounted on a second table $20 \mathrm{in}$. high. An alcove with a 10-in. diameter was cut from the center of the infant work table. The infants were seated in a specially designed chair, similar to commercial infant seats, and placed in the alcove to view the display. The surface of the infant table was about shoulder high for the youngest infants.

The visual display was made of two

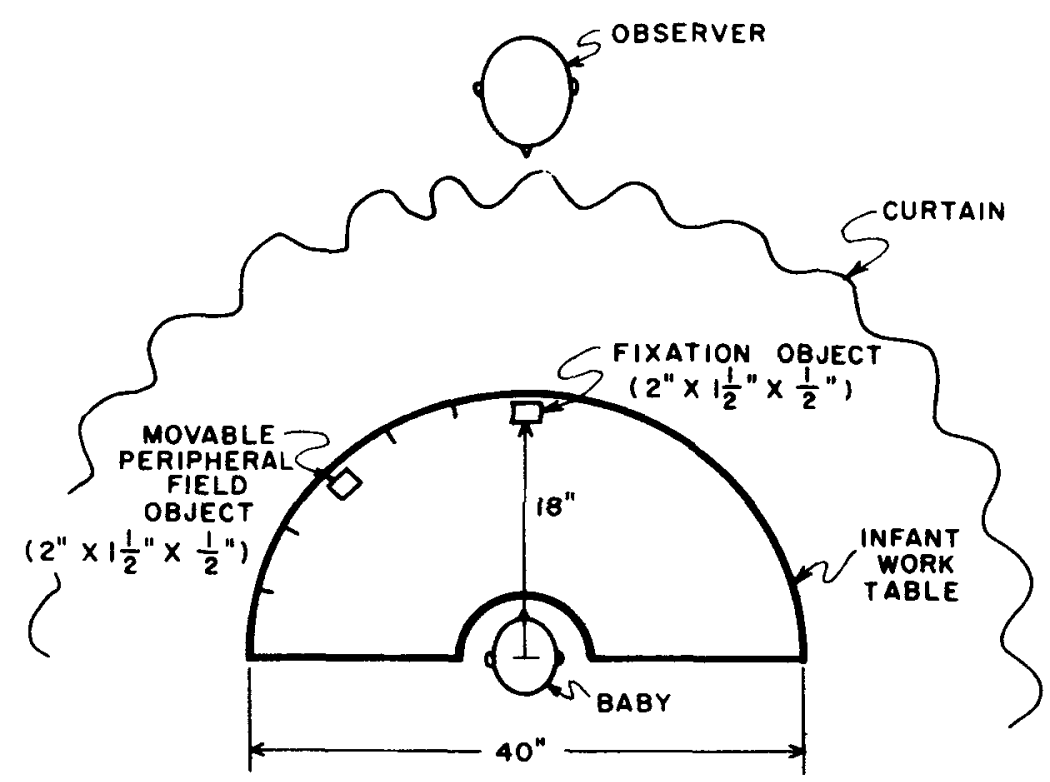

Fig. 1. Apparatus and viewing field used in the experiment viewed from above. The $O$ is behind the curtain, and an assistant is below the work table, placing the peripheral object in various radial positions. 


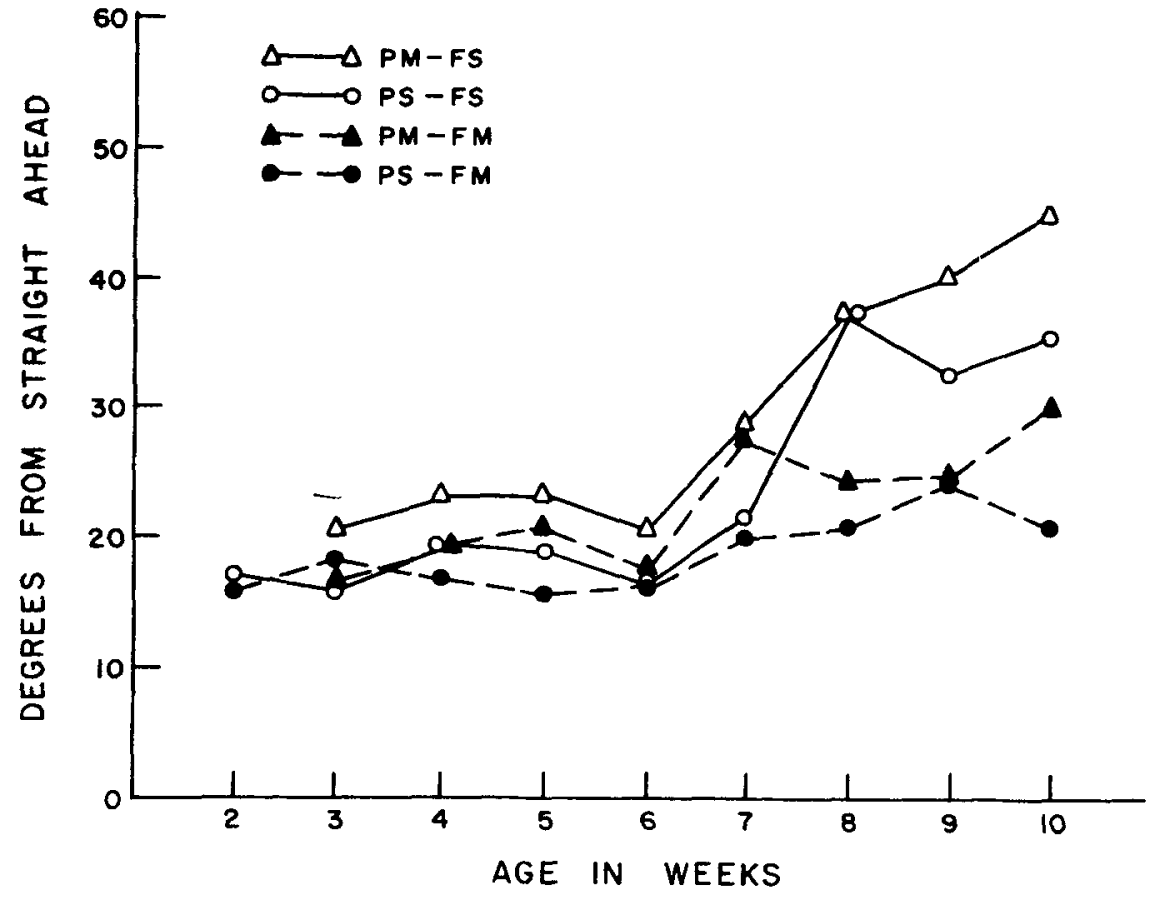

Fig. 2. The growth in the effective visual field for each week and each stimulus condition. Each point is an a verage of the limens for all $S s$ in that condition. Note how the growth in the size of the effective visual field is related to the stimulus conditions ( $P$-peripheral object; $F$-fixation object; S-object stationary; $M-$ object in motion).

position of the peripheral object. If the infant remained quiet and alert, he would continue through a Latin-square ordering of the other possible fixation- peripheral-object motion combinations. Any signs of fussing and tretting were handled by calming the infant, and, if that failed, the experiment was terminated. The same order of presentation was followed in each session for each infant.

A trial did not begin until the infant was centered, i.e., looking at the fixation object, and was terminated if the infant began to look around the field in a manner unrelated to the objects presented. When the $O$ judged that the infant was centered, the assistant brought the peripheral object up from below the infant table surface using the jacknife motion. The fixation object remained in position

Table 1

The Standard Deviation of the Effective Vieual Field (in Dearees) for Each Week by Each Stimulus Condition

\begin{tabular}{rrrrr}
\hline Week & PM-FS & PM-FM & PS-FS & PS-FM \\
\hline 2 & & & 4.1 & 3.0 \\
3 & 5.4 & 4.8 & 5.4 & 4.7 \\
4 & 7.0 & 10.4 & 10.5 & 7.6 \\
5 & 8.1 & 5.4 & 5.3 & 4.9 \\
6 & 12.0 & 4.5 & 4.6 & 3.6 \\
7 & 9.5 & 9.1 & 12.0 & 6.4 \\
8 & 12.4 & 10.8 & 11.9 & 14.6 \\
9 & 16.0 & 10.1 & 12.7 & 8.7 \\
10 & 15.6 & 13.5 & 8.2 & 10.6 \\
\hline
\end{tabular}

effects of the stimulus conditions are presented in Fig. 2, and the standard deviations about these points are presented in Table 1. Initially, the infant's effective visual field is not much greater than 15 to $20 \mathrm{deg}$ to either side of his line of regard. The initial size changes drastically with age and in relation to stimulus movement. When the fixation object is in motion and the peripheral object is stationary (PS-FM), the size of the field changes only slightly over the first 10 weeks. In contrast, when the peripheral object is in motion and the fixation object is stationary (PM-FS), the visual field at 10 weeks is 20 deg larger than it was initially and more than $20 \mathrm{deg}$ larger than it is in the PS-FM condition. The greater size of the field in the PM-FS condition was true for the group at each week studied and for 13 out of 14 cases in which an infant received both conditions in the same session. The other two conditions, both objects in motion (PM-FM) and both objects stationary (PS-FS), fall between the two extremes. It is also the case that the area of the effective visual field remains approximately the same until about 6 weeks of age, at which time its expansion begins.

Figure 3 presents the amount of time it took for the infants to look at the peripheral object at various radial directions in the different conditions.

during the entire presentation of the peripheral object. Without. its presence, the infant's gaze was found to shift haphazardly around the field, making assessment of the effective visual field impossible.

The infant was allowed $15 \mathrm{sec}$ to look at the peripheral object in all of the conditions. Fifteen seconds is an extremely long time period for the detection of a peripheral event to take place, and pilot work indicated that it was longer than even infants require. The criteria of a look were a definite shift in gaze to the peripheral object and a brief fixation of it. The judgments of different Os of when a look occurred were in agreement almost $100 \%$ of the time. If the infant did look, the time in seconds was noted and a new trial begun, with the peripheral object moved 10 deg farther away from straight ahead (and its previous position). If the infant failed to look, the peripheral object was moved $10 \mathrm{deg}$ closer to the straight-ahead position. This is essentially a method of staircase psychophysics (Guilford, 1954). The starting point was always $20 \mathrm{deg}$ from straight ahead, and two sets of three success-fail changes (absolute limens) were collected on both the left and right sides.

\section{RESULTS}

The areal size of the infant's visual field and its growth with age and the

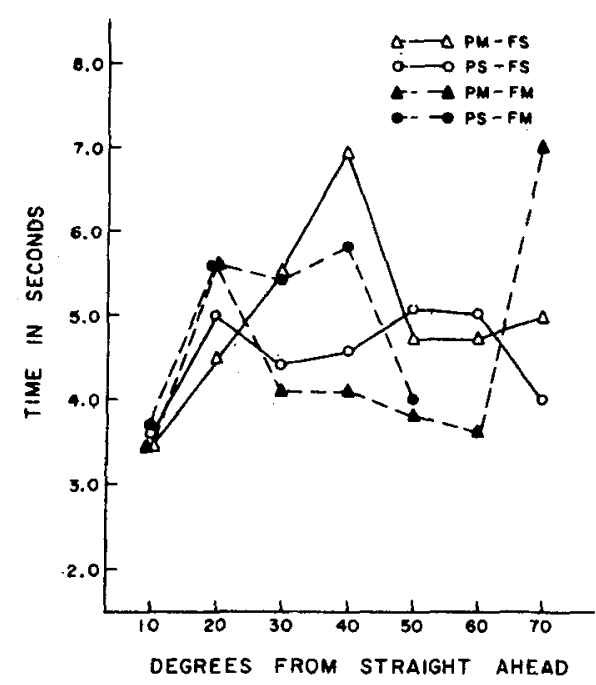

Fig. 3. The average time to look toward a given radial position of the peripheral object. No apparent relationship appears to exist between radial position and the time it took an $S$ to look from the fixation object to the peripheral object. Failure trials, when the $S$ did not look to the peripheral object within 15 sec, were excluded (P-peripheral object; F-fixation object; $S$-object stationary; M-object in motion). 


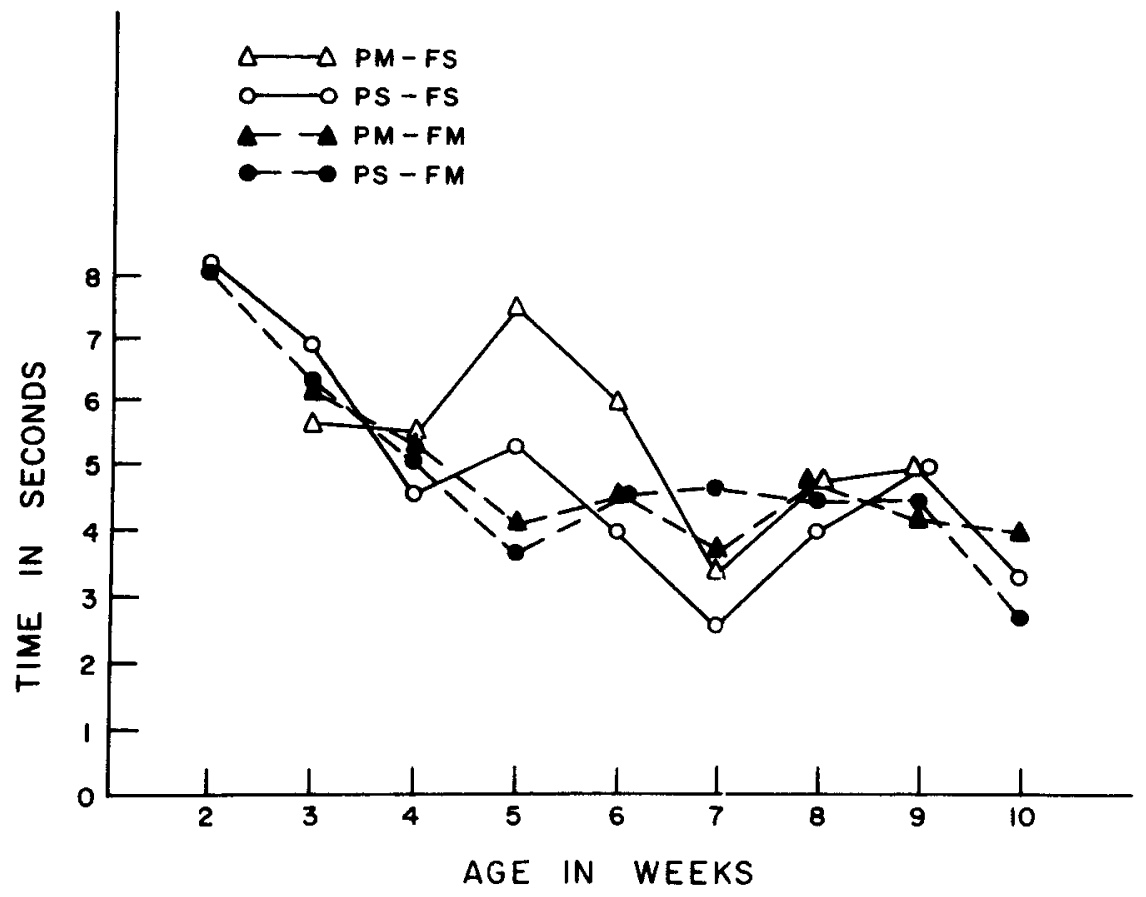

Fig. 4. Time to look toward the peripheral object in seconds for each stimulus condition for each week. There appears to be a general decrease in the time to look, but it is unrelated to the stimulus conditions ( $P \longrightarrow$ peripheral object; F-fixation object; $S-$ object stationary ; $M-$ object in motion ).

The standard deviations about the points varied between 1.1 and 3.2 sec. It is apparent that there is no relationship between time to look and radial extent. However, as the infants increased in age, the time it took them to look toward an object in the periphery decreased for all stimulus conditions as tested by a median split of time to look for Weeks 3-6 against Weeks 7-10 (Fisher exact test, $\mathrm{p}<.025$; see Fig. 4). Qualitatively, another change that took place with age was that by 10 or 11 weeks, infants did not stay fixated at the center object but continually looked away toward other points in the field. This prevented further quantification of the visual field.

\section{DISCUSSION}

The infant's effective visual field is directly related to the nature of events available for registration. Motion is a more effective producer of attention-more easily registered in the peripheral field, more compelling in the focal field. Initially, the field is quite small, but motion is already more effective in either the periphery or at the center of the field. With increasing age, the areal limits increase, but only in relation to the stimulus conditions. An increase in acuity does not appear to be a sufficient explanation. The infant's visual acuity does improve with age (Haynes, White, \& Held, 1965), but even for the

That the of head and eyes is evidenced by the general decrease in the time to look with increasing age for all target positions (cf. Tronick \& Clanton, 1971). However, that explanation would require a positive relationship to exist between radial distance and time to look, and it has nothing to say about the differential effectiveness of the stimulus conditions. Fatigue or habituation are excluded, given the use of the Latin-square design which resulted in some Ss showing their largest effective field late in the experiment.

Thus, the areal limits of the effective visual field, and their growth, reflect both stimulus control and growing functional capacity. This change in the effective visual field emphasizes that Es must consider these changes in the design of infant experiments. For example, the literature on infant's visual preferences for stimuli of different complexity levels, turns, faces, or whatever, is at best, confusing, especially for the newborn period (Gibson, 1969). Part of the problem may be that all definitions of the stimuli implicitly involve area, but none of the definitions have included an assessment of the effective areal limits of the infant's visual field. This is also supported by Salapatek and Kessen's (1966) finding that infants spend most of their time looking at a limited portion of a configuration.

Brennan (1965) described a change from an obligatory to a voluntary looking pattern in infants. Mundy-Castle and Anglin (1969) noted a similar change from a fixated to an exploratory looking pattern. Both interpreted the change as a transition from one stage of ability to another, a structural or qualitative change. However, another interpretation based on this data is that there is a quantitative growth in the effective visual field. The growth makes previously ineffective portions of the field available to be looked at.

In each of these cases, events outside the infant's effective visual field are not registered. The limits and stimulus control effects found for radial direction are also found for distance as well (Ling, 1942). It is as if the infant's effective visual field was "cone" shaped, with limits in all directions varying with stimulus conditions and expanding with development. Even though the infants appear to be able to perceive depth, for example the approach of an object (Ball \& Tronick, 1971), the limits of their effective field of vision would produce severe constraints on their ability to structure the visual world (Gibson, 1966).

\section{REFERENCES}

ARONSON, E., \& TRONICK, E. Perceptual capacities in early infancy. In J. Eliot (Ed.), Human development and cognitive processes. New York: Holt, Rinehart, \& Winston, 1970.

BALL, W., \& TRONICK, E. Infant responses to impending collision: Optical and real. Science, 1971, 171, 818-820.

BRENNAN, W., AMES, E. W., \& MOORE, $R$. W. Age differences in infants' attention to patterns of different complexity. Science, 1966, 151, 345-346. GIBSON, J. The senses considered as perceptual systems. New Yodk: Houghton-Mifflin, 1966 .

GIBSON, W. Principles of perceptual learning and development. New York: Appleton-Century-Crofts, 1969.

GUIL FORD, J. P. Prychometric methods. New York: McGraw-Hill, 1954.

HAYNES, H., WHITE, B. L., \& HELD, R. Visual accommodation in human infants. Science, 1965, 148, 528-530.

KOFFKA, K. The growth of the mind. Totowa, N.J: Littlefield \& Adams, 1959.

LING, B. C. Genetic study of sustained visual fixation and associated behavior in the human infant from birth to six months. Journal of Genetic Psychology, 1942, 61, 227-277.

MACKWORTH, N. H., \& MORANDI, A. J. Outstanding visual stimuli dominate Psychophysics, 1967, 2, 547-552. looking and scaling choices. Perception \& 
MUNDY-CASTLE, A., \& ANGLIN, J. M. Looking strategies in infants. Paper presented at Society for Research in presented at Society for Research in Barbara, 1969.

PEIPER, A. Cerebral function in infancy and childhood. New York: Consultants
Bureau, 1963.

SALAPATEK, $P_{\bullet} \&$ KESSON, $W$. Visual scanning of triangles by the human newborn. Journal of Experimental Child Psychology, 1966, 3, 155-167.

TREVARTHEN, C. Two mechanisms of vision in primates. Psychologische
Forschung, 1968, 31, 299-337.

TRONICK, E., \& CLANTON, C. Infant looking patterns. Vision Research, 1971 11, 1479-1486.

(Received for publication November 24 1971.) 